\title{
A Public Database on Traumatic Brachial Plexus Injury
}

3 Cristiane B Patroclo ${ }^{1,2} *$, Bia L Ramalho ${ }^{1,2}$, Juliana S Maia ${ }^{1,2}$, Maria Luíza Rangel ${ }^{1,2}$, Fernanda

4 F Torres $^{1,2}$, Lidiane Souza1,2, Kelly R Braghetto ${ }^{3}$, Claudia D Vargas ${ }^{1,2}$.

$5 \quad$. Laboratory of Neurobiology of Movement, Institute of Biophysics Carlos Chagas Filho, Federal

6 University of Rio de Janeiro, Rio de Janeiro, Brazil

$7 \quad 2$. Laboratory of Neuroscience and Rehabilitation, Institute of Neurology Deolindo Couto, Federal

8 University of Rio de Janeiro, Rio de Janeiro, Brazil

$9{ }^{3}$. Department of Computer Science, Institute of Mathematics and Statistics, University of São Paulo,

10 São Paulo, Brazil

$11 *$ Correspondence:

12 Cristiane B Patroclo

13 crispatroclo@yahoo.com.br

Keywords: public database, brachial plexus, traumatic injury, sharing data, traffic injury

\section{Abstract}

We hereby present the first worldwide public digital database centred on adult Traumatic Brachial Plexus Injury (TBPI). This initiative aims at reducing distance between clinical and experimental practice and encouraging data sharing and reuse. Detailed electronic questionnaires made with the free software LimeSurvey were designed to collect patients' epidemiological, physical and clinical data. The freely available software Neuroscience Experiments System (NES) was employed to support data storage and management. First results of this effort concern data collected from 109 Brazilian adult TBPI patients with varying degrees of functional impairment. The sample is composed by large majority of men $(84.4 \%)$, mean age of 32.1 (11.3 SD) years old, victims of motorcycle accidents $(67 \%)$. The similarity of this dataset basic descriptors with those from previous reports in TBPI validates the strategies employed herein. Managing data from diverse provenance in TBPI may allow identifying functional markers related to the patients' clinical improvement and foster the development of new investigative tools to unveil its mechanisms. 


\section{Introduction}

Construction, maintenance and curation of public databases are becoming fundamental to propel our understanding of the nervous system function and dysfunction. This data-sharing paradigm emerged in the literature and media in the $90 \mathrm{~s}^{(1,2)}$ with the International Consortium for Brain Mapping, the first major data sharing initiative for fMRI measures. The growth of this trend had only been possible by the technological progress that substantially increased the capacity to generate data in neuroscience and the huge development of information technology ${ }^{(3,4)}$. In accordance to the data-sharing paradigm, we devised a public database able to store data of diverse provenance in the domain of traumatic brachial plexus injury (TBPI) and its surgical reconstruction in adult patients.

The brachial plexus is a nerve net that congregates the ventral divisions from C5 to T1 spinal nerves and is responsible for ipsilateral upper limb motricity and sensibility ${ }^{(5)}$. TBPI mainly affects young males usually involved in motorcycle accidents, often leading to severe motor and sensitive impairment in the affected upper $\operatorname{limb}{ }^{(6)}$. Psychic, social and quality of life impairments are also reported $^{(7,8)}$.

TBPI's treatment of choice is the surgical reconstruction allied with physical therapy ${ }^{(9,10)}$. Muscle strength is by far the focus of most frequent treatment outcomes ${ }^{(11,12)}$.

TBPI main associated prognostic factors are: patient's age, lesion site, severity and mechanisms, associated traumatic lesions, time interval between the injury occurrence and surgery and the employed surgical repair technique ${ }^{(13-23)}$. However, the lesion complexity, its heterogeneity, and the well documented brain plasticity that follows a peripheral nerve injury ${ }^{(24-26)}$, make it very difficult to preview patients' outcomes. This argues for the incorporation of other measures of success after TBPI ${ }^{(12)}$ and for the development of specific instruments for the functional evaluation of these patients ${ }^{(27)}$.

In this context, for the first time a set of detailed questionnaires designed to collect TBPI patients' epidemiological, clinical, physical and surgical data together with the first set of anonymized results concerning 109 TBPI patients are made publicly available, as described here.

\section{$2 \quad$ Methods}

The development of the TBPI database was carried out by a multidisciplinary team comprising physicians, physiotherapists, neuroscientists and computer scientists.

Database building efforts involved the following steps: 2.1 patients selection, 2.2 data selection, 2.3 electronic questionnaires development, 2.4 data management platform development, 2.5 data entering, 2.6 de-identification of personal data, 2.7 data access. 


\subsection{Patient selection}

All the TBPI patients included in the database were older than 18 years and were evaluated at the Institute of Neurology Deolindo Couto of the Federal University of Rio de Janeiro (INDC-UFRJ) from 2010 to 2017 by physicians and physical therapists. Patients prospectively evaluated voluntarily gave their written consent allowing the publication of their de-identified data in a public database. Retrospective data, collected by the same group before the TBPI Database project, was also allowed to be included in the database by the local Ethics Committee.

\subsection{Data selection}

Epidemiological, clinical, physical and surgical data were collected through specifically designed questionnaires named: Unified Admission Assessment, Unified Follow up Assessment and Unified Surgical Evaluation.

Unified Admission Assessment (UAA) concerns information about TBPI (side, causes and associated lesions), ongoing treatments (physiotherapy, orthosis, medication), neurological exam with visual inspection (glenohumeral subluxation, scoliosis, Horner's syndrome, swelling, scares and trophic changes), presence of Tinel sign, sensory evaluation (light touch, pain, joint position sense, kinesthesia and pallesthesia), motor evaluation (motion range and muscle strength), pain occurrence and lesion site (preferably based on surgical information, followed by complementary exam, previous notes and physical exam). UAA was filled at the first interview and relied on medical records for missing data.

Unified Follow up Assessment (UFA) reviews ongoing treatments and neurological exam previously evaluated with UAA. It was filled at follow-up visits, ideally at each six months.

Unified Surgical Evaluation (USE) details surgical findings (level and type of lesion) and the employed procedures (type of surgery with its specificities). The USE questionnaire is filled by the neurosurgeons just after the surgery or by the researchers based on medical records.

\subsection{Electronic questionnaires development}

Electronic questionnaires present advantages when compared to paper-based ones such as ease and speed of administration, enforcement of data standardization (e.g., by using fixed choice response formats), immediate connection with the database, easier access to data and efficiency and security in data storage ${ }^{(28)}$. The questionnaires described in the previous section were created in an electronic format using the open-source survey system LimeSurvey. The decision for choosing LimeSurvey stemmed from the free availability of the tool and the fact that it relies on an underlying database management software, which can be deployed on a server that is deemed appropriate to store the target data and customized to support different data access policies. In addition, it supports several question types, enables the definition of restrictions on questions, admits design of logical branching based on answers and scores, allows the creation of multilingual surveys, enables the user to export collected data into spreadsheets, and supports general survey security settings. 
107

108

109

110

111

112

113

114

115

116

117

118

119

120

121

122

123

124

125

126

127

128

129

130

131

132

133

134

135

136

137

138

139

140

141

142
The created electronic questionnaires follow the general structure of the paper-based questionnaires previously used by the group, facilitating adherence. The UAA, UFA and USE questionnaires favor multiple-choice questions to avoid excessive answers variability and enforce standardization in data entering. Besides, conditional branching arrangement of questions were employed to ensure data detailing.

\subsection{Data management platform development}

To meet the TBPI project's demands for data acquisition, management and sharing, a software tool named Neuroscience Experiment System (NES) was developed. NES is a free, safe, user-friendly platform originally devoted to assist researchers in their experimental data collecting routine and to enable experiments reproduction. The platform keeps comprehensive and detailed descriptions of experimental protocols in a unified repository, as well as data and metadata from different provenance, including epidemiological, clinical and physical database described in the present report. With this aim, NES was integrated with LimeSurvey to facilitate questionnaires' administration and to centralize data access.

\subsection{Data entering}

Prospective TBPI patients' data were collected through personal interview conducted by a neurologist and/or a physiotherapist. The collected data was directly registered in the database through the NES platform. Retrospective TBPI patients' data required careful selection, cleaning and transformation before entry the database. This process was done by the same neurologist and/or a physiotherapist who collected prospective data. Also, different sources were used in a hierarchical way to ensure data completeness and consistency: previous research records, medical records and patients' report via phone call.

\subsection{De-identification of personal data}

Patients' identification is only known by the local facility researchers. For the public version of the database the following information are suppressed: name; birthdate; ethnicity; address; phone number; professional information; dates (replaced by time intervals); injury circumstances as local of occurrence and details of hospitalization; and surgical and treatment places. Each patient in the database is identified by a noninformative code automatically generated by NES. These safety actions were inspired by the "Guidelines for working with small numbers" ${ }^{(29)}$ from the Washington State Department of Health and by Hrynaszkiewicz $(2010)^{(30)}$. 


\subsection{Data access}

The anonymized version of the TBPI database (exported from NES) is available to public access in an Open Database portal https://neuromatdb.numec.prp.usp.br/experiments/brachial-plexusinjury-database-v2/. Periodic database updates will be available in the portal with a brief description of the changes in the updated data as compared to the previous version.

The data are also hosted at figshare.

All public experimental data in the NeuroMat Open Database is available under the Creative Commons Attribution 4.0 International (CC BY 4.0) (https://creativecommons.org/licenses/by/4.0/) license. Therefore, to be able to download data, a potential user is required to agree and accept the CC BY 4.0 terms. According to CC BY 4.0, licensees may copy, distribute, and display the data and generate derivative works based on it only if they give the author(s) the appropriate credits (attribution).

\section{Code availability}

NES version 1.39 was used to create and manage the BPTI database. NES is a free software https://github.com/neuromat/nes.

LimeSurvey version 2.05 was used to create and manage the electronic questionnaires. Its source code and documentation are available at http://www.limesurvey.org/. The structures of imported in Limesurvey platform to (re)create a questionnaire.

\section{$4 \quad$ Data Records}

No specific software is required to handle the TBPI data from the NeuroMat Open Database. The dataset is downloaded as a single .ZIP file. It compresses several directories with plain-text files

168 containing textual and numeric data (e.g., .CSV files for tabular data and .JSON files for metadata) 169 that can be opened with a large variety of computational tools, from simple text editors to general170 purpose statistical softwares.

171 In the root directory of the decompressed dataset, the data and metadata files are organized 172 according the following hierarchical structure:

173 - Citation.txt 
$178+$ Experimental_protocol (directory)

- unified-admission-assessment.pdf

- unified-surgical-evaluation.lss

- unified-surgical-evaluation.pdf

- Fields_Q44071_en.csv

- Fields_Q44071_pt-BR.CSV

- Fields_Q61802_en.csv

- Fields_Q61802_pt-BR.Csv

- Fields_Q92510_en.csv

- Fields_Q92510_pt-BR.CSV 
+ Q92510_unified-follow-up-assessment (directory)

- Responses_Q92510.CsV

+ Step_2.1_questionnaire (directory)

- Q61802_unified-surgical-evaluation.csv

+ Step_2.2_questionnaire (directory)

Citation.txt and License.txt contain information about the license under which the dataset has been published and how it must be cited.

Experiment.csv is a CSV (comma-separated values) file with basic information (name,

In the Questionnaire_metadata subdirectory, one may found .CSV files which describe the complete structure of the three questionnaires both in English and Brazilian Portuguese. Each .CSV 
file contains, for each question in the questionnaire, its identifying code, description (complete text), type, sub-questions and answer options. To facilitate the understanding and manipulation of data collected using the questionnaires, we adopted a naming convention to question codes: the prefix of the code indicates the type of data collected by the question. For example, for the question "How old is the patient?", intAge should be used as code. The "int" prefix indicates that the value for age is an integer number. Table 1 shows the data types of the questions which appear in our questionnaires and their respective code prefixes.

The patients' data collected by means of the electronic questionnaires is replicated in two directories: Per_questionnaire_data and Per_participant_data. In the former, for each one of the three questionnaires, there is a directory containing a .CSV file (e.g., Responses_Q44071.csv) with all the patient responses collected through the questionnaire. Each line in these CSV files contains a response for a particular patient; the identification of the patient appears in the column entitled "participant_code". Each patient has exactly one response for the Unified Admission Assessment, but he/she can have none or more responses for the Unified Surgical Evaluation and Unified Follow-up Assessment. In other words, the participant code is unique in the Responses_Q44071.csv file, but not in Responses_Q61802.csv and Responses_Q92510.csv. Different lines in the .CSV file with a same participant code indicate different responses of a same patient for a same questionnaire.

In the Per_participant_data directory, there is directory for each patient who participated in the study, containing a .CSV file for each questionnaire filled for him/her. If a patient has multiple responses for a given questionnaire, they will appear as different lines in its .CSV file. Each CSV file in the Per_participant_data directory contains responses of only one patient.

In the .CSV file containing responses for a given questionnaire, each column corresponds to a question or a sub-question of the questionnaire. All information required to understand the meaning of a column and its values can be recovered from the questionnaire's metadata file through the column name. Searching by a response column name in the "question_index" column of the questionnaire's metadata file, one will find one or more lines which describe the correspondent question or subquestion and the type of responses it accepts.

\section{$5 \quad$ Technical Validation}

To our knowledge, this is the first public dataset concerning adult TBPI, thus precluding its comparison with other databases. Therefore, in this section we will limit our data validation to the basic epidemiological descriptors available in literature.

The present dataset contains data from 109 Brazilian adult patients with TBPI. In agreement with the classical report of Narakas ${ }^{(17)}$, and subsequent national ${ }^{(31-34)}$ and international series ${ }^{(19,20,35)}$, our sample is composed mainly by young (average age at injury: 32.1 years $\pm 11.3 \mathrm{SD}$ ) male $(84.4 \%$ ) subjects involved in traffic accidents. Fifty-six patients $(51 \%)$ have left side injury. Figure $1 \mathrm{~A}$ depicts results of TBPI causes in 114 nerve injuries from 109 patients (one patient had bilateral injury and four patients had unilateral injury provoked by two different causes). The entire brachial plexus (encompassing C5 to T1 roots) is affected in $44 \%$ of the patients. Four patients present more than one site of lesion (Figure 1B). 
The percentage of associated injuries (fractures and traumatic brain, spinal cord and vascular injuries) and reported pain in the 109 patients (Figure 1C) are also in accordance with the literature ${ }^{(14,31-33,36,37)}$.

The dataset also includes information of 81 UFA from 46 patients (42\%) and 44 USE from 28 patients. The average number of surgical reconstruction procedures per patient is $1.8 \pm 0.6 \mathrm{SD}$, with the nerve transfer $(77 \%)$ as the most frequent.

Differing from published series, which limit their focus to specific aspects of TBPI, our database provides in the same set a wide range of information concerning this injury. This provides the opportunity of exploring the combination of these descriptors envisioning its deeper understanding.

In conclusion, this is the first report on a public database on TBPI. This is a unique initiative, resulting from a multidisciplinary effort, that stands out by its richness in clinical and epidemiological data and sharing potential. The similarity of this data with other national and international series endorses the quality of the present dataset. TBPI results mainly from traffic accidents, a huge public health problem especially in developing countries, and affects working age population with disabling consequences. In this sense, improving the knowledge on the TBPI can contribute to patient care, support governmental health strategies and give insights into factors related to its prognosis.

\section{$6 \quad$ Conflict of Interest}

The authors declare that the research was conducted in the absence of any commercial or financial relationships that could be construed as a potential conflict of interest.

\section{$7 \quad$ Author Contributions}

CBP: questionnaires' creation, questionnaires' translation, data collection, data analysis, writing, reviewing

BLR: questionnaires' creation, questionnaires' translation, data collection, data analysis, writing, reviewing

JM: questionnaires' creation, data collection, data analysis

MLR: questionnaires' creation, questionnaires' translation, data collection

FFT: questionnaires' translation, reviewing

LS: questionnaires' creation, data collection

KRB: intellectual conception, data analysis, writing, reviewing 
311 CDV: intellectual conception, questionnaires' creation, data analysis, writing, reviewing

312

3139 Funding

314 This work is part of the ABRAÇO Initiative for the Brachial Plexus Injury 315 (http://abraco.numec.prp.usp.br/) of the Fundação de Amparo à Pesquisa do Estado de São 316 Paulo (FAPESP)'s Research, Innovation and Dissemination Center for Neuromathematics (grant 317 2013/07699-0, http://neuromat.numec.prp.usp.br/). It was also supported by the Conselho Nacional 318 de Pesquisa (CNPq) (grants 306817/2014-4, 426579/2016-0 and 309560/2017-9) and the Fundação 319 de Amparo à Pesquisa do Rio de Janeiro FAPERJ (grants E-26/111.655/ 2012, E26/010.002902/2014 320 and E-26/010.002474/2016; CNE 202.785/2018).

\section{List of abbreviations:}

323 INDC-UFRJ: Institute of Neurology Deolindo Couto of the Federal University of Rio de Janeiro

324 NES: Neuroscience Experiment System

325 TBPI: traumatic brachial plexus injury

326 UAA: Unified Admission Assessment

327 UFA: Unified Follow up Assessment

328 USE: Unified Surgical Evaluation

\section{Acknowledgments}

We would like to thank José Fernando Guedes Correa, Paulo Leonardo Tavares, José Vicente

\section{References}

336 1. Chicurel M. Databasing the brain. Nature (2000) 406:822-825. DOI: 10.1038/35022659

337 2. Koslow SH. Should the neuroscience community make a paradigm shift to sharing primary 338 data? Nat. Neurosci. (2000) 3:863-865. DOI: 10.1038/78760

339 3. Bouchard KE, Aimone JB, Chun M, Dean T, Denker M, Diesmann M, et al. High- 
Performance Computing in Neuroscience for Data-Driven Discovery, Integration, and Dissemination. Neuron (2016) 92:628-631. DOI: 10.1016/j.neuron.2016.10.035

4. Akil H, Martone ME, Van Essen DC. Challenges and opportunities in mining neuroscience data. Science (2011) 331:708-712. DOI: 10.1126/science.1199305

344 5. Russel SM. Examination of Peripheral nerve injuries: an anatomical approach. New York:

6. Tung TH, Mackinnon, SE. Brachial plexus injuries. Clin. Plast. Surg. (2003) 30:269-287.

7. Mancuso CA, Lee SK, Dy CJ, Landers ZA, Model Z, Wolfe SW. Expectations and limitations due to brachial plexus injury: a qualitative study. Hand (2015) 10:741-749. DOI: 10.1007/s11552-015-9761-Z

8. Franzblau L, Chung KC. Psychosocial outcomes and coping after complete avulsion traumatic brachial plexus injury.

9. Chuang DCC. Adult Brachial Plexus Reconstruction with the Level of Injury: Review and

Personal Experience. Plast. Reconstr. Surg. (2009) 124:e359-e369. DOI: 10.1097/PRS.0b013e3181bcf16c.

10. Gordon T, English AW. Strategies to promote peripheral nerve regeneration: Electrical

11. Bengtson KA, Spinner RJ, Bishop AT, Kaufman KR, Coleman-Wood K, Kircher MF, et al. Measuring Outcomes in Adult Brachial Plexus Reconstruction. Hand Clin. (2008) 24:401415. DOI: 10.1016/j.hcl.2008.04.001

12. Dy CJ, Garg R, Lee SK, Tow P, Mancuso CA, Wolfe SW. A systematic review of outcomes

13. Ricardo M. Surgical treatment of brachial plexus injuries in adults. Int. Orthop. (2005) reporting for brachial plexus reconstruction. J. Hand Surg. Am. (2015) 40:308-313. DOI: 10.1016/j.jhsa.2014.10.033

14. Siqueira MG, Martins RS. Surgical treatment of adult traumatic brachial plexus injuries: An

15. Flores LP. The importance of the preoperative clinical parameters and the intraoperative electrophysiological monitoring in brachial plexus surgery. Arq. Neuropsiquiatr. (2011) 69:654-659 
17. Narakas AO. The treatment of brachial plexus injuries. Int. Orthop. (1985) 9:29-36. DOI: $10.1007 / \mathrm{bf} 00267034$

18. Garg R, Merrell GA, Hillstrom HJ, Wolfe SW. Comparison of nerve transfers and nerve grafting for traumatic upper plexus palsy: A systematic review and analysis. J. Bone Joint Surg. Am. (2011) 93:819-829. DOI: 10.2106/JBJS.I.01602

19. Merrell GA, Barrie KA, Katz DL, Wolfe SW. Results of nerve transfer techniques for restoration of shoulder and elbow function in the context of a meta-analysis of the English literature. J. Hand Surg. Am. (2001) 26:303-314. DOI: 10.1053/jhsu.2001.21518

20. Terzis JK., Verkris MD, Soucacos PN. Outcomes of brachial plexus reconstruction in 204 patients with devastating paralysis. Plast. Reconstr. Surg. (1999) 104:1221-1240. DOI: 10.1097/00006534-199910000-00001

21. Thatte MR, Babhulkar S, Hiremath A. Brachial plexus injury in adults: Diagnosis and surgical treatment strategies. Ann Indian Acad Neurol (2013) 16:26-33. DOI: 10.4103/09722327.107686

22. Ahmed-Labib M, Golan JD, Jacques L. Functional outcome of brachial plexus reconstruction after trauma. Neurosurgery (2007) 61:1016-1023. DOI: 10.1227/01.neu.0000303197.87672.31

23. Yang LJS, Chang KWC, Chung KC. A systematic review of nerve transfer and nerve repair for the treatment of adult upper brachial plexus injury. Neurosurgery (2012) 71:417-429. DOI: 10.1227/NEU.0b013e318257be98

24. Reilly KT, Sirigu A. The Motor Cortex and Its Role in Phantom Limb Phenomena. Neurosci. (2008) 14:195-202. DOI: 10.1177/1073858407309466

25. Vargas CD, Aballéa A, Rodrigues EC, Reilly KT, Mercier C, Petruzzo P, et al. Re-emergence of hand-muscle representations in human motor cortex after hand allograft. Proc. Natl. Acad. Sci. U. S. A. (2009) 106:7197-202. DOI: 10.1073/pnas.0809614106

26. Lundborg G. Nerve injury and repair - a challenge to the plastic brain. J. Peripher. Nerv. Syst. (2003) 8:209-226. DOI:10.1111/j.1085-9489.2003.03027.x

27. Hill BE, Williams G, Bialocerkowski AE. Clinimetric evaluation of questionnaires used to assess activity after traumatic brachial plexus injury in adults: A systematic review. Arch. Phys. Med. Rehabil. (2011) 92:2082-2089. DOI: 10.1016/j.apmr.2011.07.188

28. Braghetto KR, Nascimento AS. Guidelines for Developing Electronic Questionnaires to Collect Experimental Data. http://neuromat.numec.prp.usp.br/relatorio/artigos/krbraghetto_asnascimento_artigo_neuromat .pdf (2014).

29. Van Eweyk J, Macdonald SC. Guidelines for Working with Small Numbers. Washington State Department of Health (2012). 
30. Hrynaszkiewicz I, Norton ML, Vickers AJ, Altman DG. Preparing raw clinical data for publication: guidance for journal editors, authors, and peer reviewers. BMJ (2010) 340:1-9. DOI: $10.1136 / \mathrm{bmj} . c 181$

DOI: $10.1136 / \mathrm{bmj} . \mathrm{c} 181$

413

31. Faglioni W, Siqueira MG, Martins RS, Heise CO, Foroni L. The epidemiology of adult traumatic brachial plexus lesions in a large metropolis. Acta Neurochir. (Wien). (2014) 156:1025-1028. DOI: 10.1007/s00701-013-1948-x

32. Mello Junior JS, Souza TCR, Andrade FG, Castaneda L, Baptista AF, Nunes K, et al. Perfil epidemiológico de pacientes com lesão traumática do plexo braquial avaliados em um hospital universitário no Rio de Janeiro, Brasil, 2011. TT - Epidemiological profile of patients with traumatic brachial plexus injury evaluated in a university hos. Rev. Bras. Neurol. (2012) 48:711.

33. Flores LP. Estudo epidemiológico das lesões traumáticas de plexo braquial em adultos. Arq.

34. de Moraes FB, Kwae MY, da Silva RP, Porto CC, Magalhães D de P, Paulino MV. Clinical aspects of patients with traumatic lesions of the brachial plexus following surgical treatment. Rev. Bras. Ortop. (2015) 50:556-561. DOI: 10.1016/j.rboe.2015.08.015

36. Moran SL, Steinmann SP, Shin AY. Adult brachial plexus injuries: Mechanism, patterns of

35. Jain DKA, Bhardwaj P, Venkataramani H, Sabapathy SR. An epidemiological study of traumatic brachial plexus injury patients treated at an Indian centre. Indian J. Plast. Surg. (2012) 45:498-503. DOI: 10.4103/0970-0358.105960

37. Ciaramitaro P, Padua L, Devigili G, Rota E, Tamburin S, Eleopra R, et al. Prevalence of Neuropathic Pain in Patients with Traumatic Brachial Plexus Injury: A Multicenter Prospective Hospital-Based Study. Pain Med. (2017) 18:2428-2432. doi:10.1093/pm/pnw360

\section{Data Availability Statement}

436 The datasets A Public Database on Traumatic Brachial Plexus Injury for this study can be found in 437 the Figshare https://figshare.com/s/6d11e763c9af85b5c95f. 
443 Table 1 - LimeSurvey question types and their respective prefix codes.

\begin{tabular}{|c|c|c|}
\hline Question Type Name & Description & $\begin{array}{l}\text { Code } \\
\text { Prefix }\end{array}$ \\
\hline \multirow[t]{2}{*}{ Numeric input } & Typing integer numbers & Int \\
\hline & Typing decimal numbers & Dec \\
\hline Free text & \multirow[t]{2}{*}{ Typing text } & \multirow[t]{3}{*}{ Tex } \\
\hline Long free text & & \\
\hline Array of multiple texts & Typing multiple texts (in an array of text boxes) & \\
\hline Date & Typing/selecting date & Dat \\
\hline List & Selecting a single option among many & \multirow[t]{2}{*}{ Lst } \\
\hline Array of lists & Selecting a single option among many in each list & \\
\hline Multiple choices & \multirow[t]{2}{*}{ Selecting one or more options among many } & \multirow[t]{2}{*}{ Mul } \\
\hline $\begin{array}{l}\text { Multiple choices with } \\
\text { comments }\end{array}$ & & \\
\hline Equation & $\begin{array}{l}\text { Value calculated from answers given to previous } \\
\text { questions }\end{array}$ & Equ \\
\hline Yes or No & Answering "yes" or "no" & Yon \\
\hline
\end{tabular}




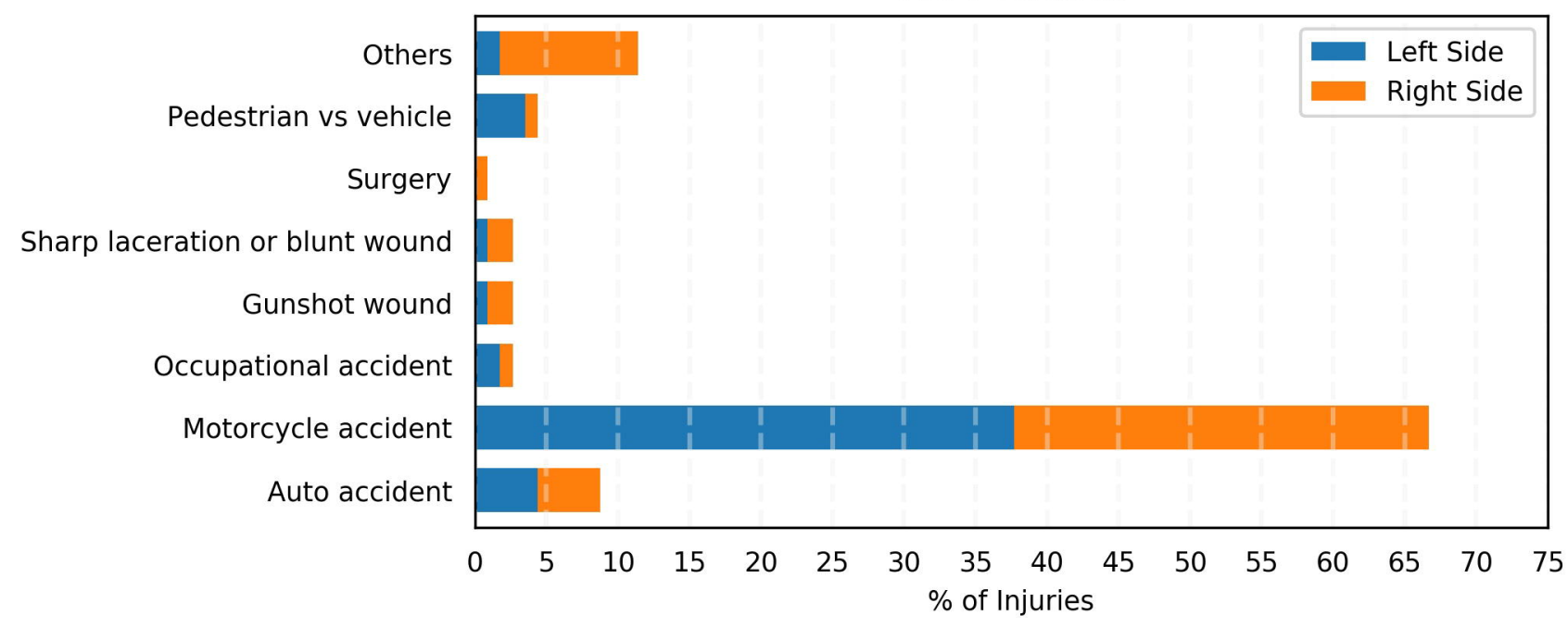

\section{TBPI Site}

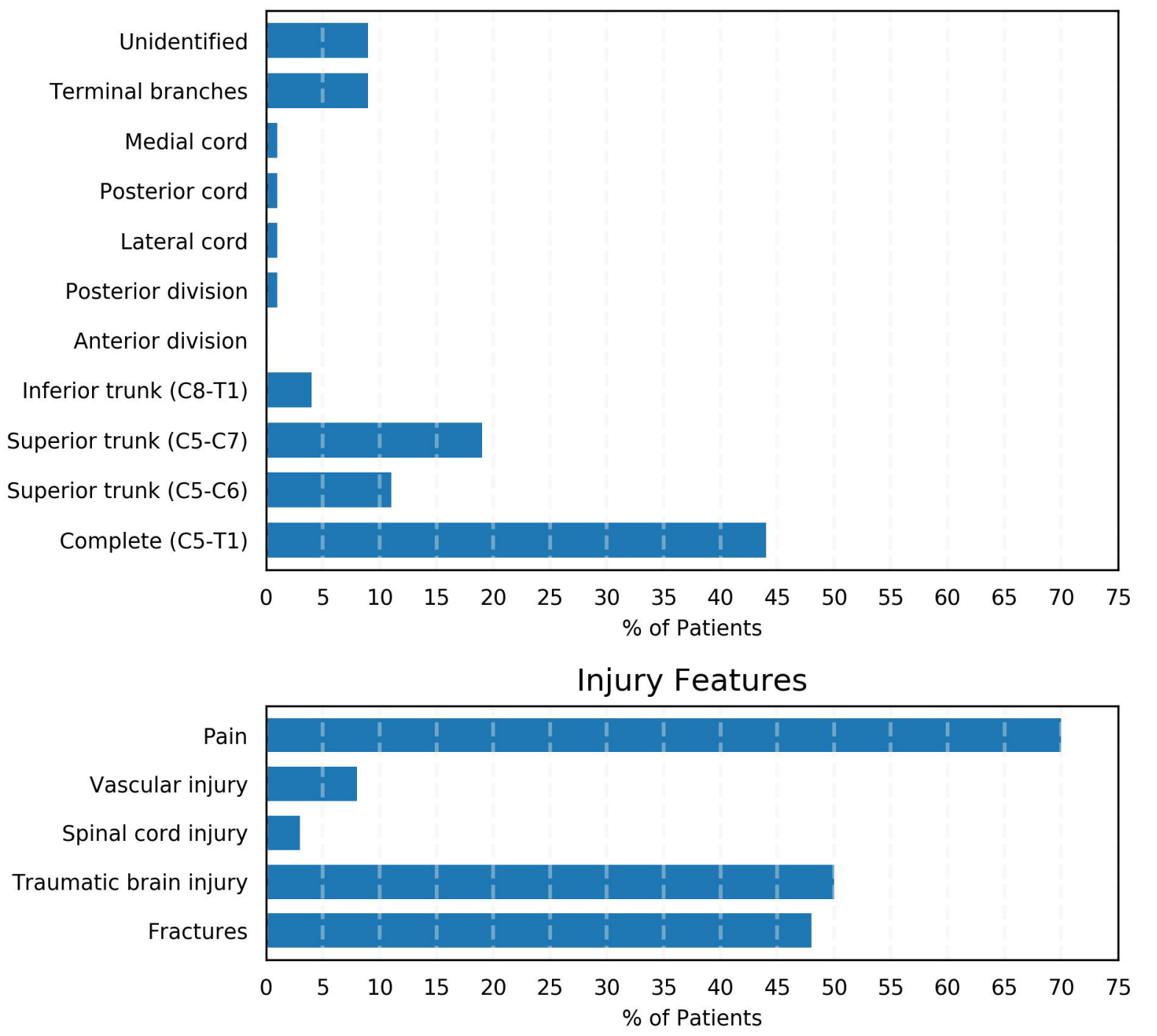

